\title{
MicroRNAs as Salivary Markers for Periodontal Diseases: A New Diagnostic Approach?
}

\author{
Gerhard Schmalz, ${ }^{1}$ Simin Li, ${ }^{1}$ Ralph Burkhardt, ${ }^{2}$ Sven Rinke, ${ }^{3}$ Felix Krause, \\ Rainer Haak, ${ }^{1}$ and Dirk Ziebolz ${ }^{1}$ \\ ${ }^{1}$ Department of Cariology, Endodontology and Periodontology, University of Leipzig, 04109 Leipzig, Germany \\ ${ }^{2}$ Institute of Laboratory Medicine, Clinical Chemistry, and Molecular Diagnostics, University of Leipzig, 04109 Leipzig, Germany \\ ${ }^{3}$ Department of Prosthodontics, University Medical Center Goettingen, Goettingen, Germany
}

Correspondence should be addressed to Dirk Ziebolz; dirk.ziebolz@medizin.uni-leipzig.de

Received 19 January 2016; Accepted 20 March 2016

Academic Editor: Kenji Yoshida

Copyright (C) 2016 Gerhard Schmalz et al. This is an open access article distributed under the Creative Commons Attribution License, which permits unrestricted use, distribution, and reproduction in any medium, provided the original work is properly cited.

\begin{abstract}
The aim of this review is to discuss current findings regarding the roles of miRNAs in periodontal diseases and the potential use of saliva as a diagnostic medium for corresponding miRNA investigations. For periodontal disease, investigations have been restricted to tissue samples and five miRNAs, that is, miR-142-3p, miR-146a, miR-155, miR-203, and miR-223, were repeatedly validated in vivo and in vitro by different validation methods. Particularly noticeable are the small sample sizes, different internal controls, and different case definitions of periodontitis in in vivo studies. Beside of that, the validated miRNAs are associated with inflammation and therefore with various diseases. Furthermore, several studies successfully explored the use of salivary miRNA species for the diagnosis of oral cancer. Different cancer types were investigated and heterogeneous methodology was used; moreover, no overlap of results was found. In conclusion, five miRNAs have consistently been reported for periodontitis; however, their disease specificity, detectability, and expression in saliva and their importance as noninvasive markers are questionable. In principle, a salivary miRNA diagnostic method seems feasible. However, standardized criteria and protocols for preanalytics, measurements, and analysis should be established to obtain comparable results across different studies.
\end{abstract}

\section{Introduction}

MicroRNAs (miRNAs) are endogenous $\sim 22 \mathrm{nt}$ RNAs that play important regulatory roles in animals and plants by targeting mRNAs for cleavage or translational repression [1]. Many multifarious investigations regarding miRNAs have demonstrated their essential roles in physiological and pathological processes in the immune response [2]. Additionally, miRNAs likely contribute to development and progression of systemic diseases, such as cancer [3] and vascular disease [4]. Consequently, there is considerable interest in use of miRNAs as not only diagnostic markers but also potential therapeutic targets for various diseases [5]. In particular, the roles of miRNAs in oral cancer and precancer have been investigated. MiRNA expression appears to differ between healthy tissue and squamous cell carcinoma tissues of the oral cavity, which is discussed in an existing review [6]. Furthermore, some investigations have linked precancerous lesions and their risk of becoming malignant to changes in miRNA expression [7].

Furthermore, miRNAs are associated with bacterial infections [8] and, thus, are most likely associated with infectious diseases of oral cavity, for example, dental caries, endodontic infections, and periodontitis. Kim et al. (2015), however, already give an overview of current findings regarding dental disease, including periodontal disease and oral cancer. Kim et al. (2015) also mentioned limitations of current diagnostic and potential benefit of salivary miRNA diagnostic [9]. This is the reason to discuss the roles of miRNAs in periodontal diseases. As of now, only a few in vitro and in vivo investigations regarding these roles are available. A recent review article demonstrated potential roles of miRNAs during periodontal inflammation, showing the considerable role of them in 
periodontitis [10]. Thereby, different roles and mechanisms are shown and a very comprehensive overview on molecular pathways is given; however, methodology was only discussed between selected studies.

As mentioned above, an interesting approach is the analysis of miRNA species in saliva. High quality miRNAs were shown to be detectable in saliva [11], and the possibility of their usage in oral cancer detection appears to have a high potential for future diagnostics [12]. This noninvasive approach could be relevant for further diseases of the oral cavity. Nevertheless, the potential usage of miRNA as salivary markers for periodontal diseases was already not discussed yet. As shown for oral cancer, using miRNA for noninvasive diagnostics seems feasible [12] and could therefore serve as a salivary marker for periodontitis. The potential is high, so maybe recent indispensable invasive diagnostics or X-ray could become avoided by salivary miRNA diagnostic in the future and an early diagnosis might help to prevent bone and, in conclusion, tooth loss.

Nevertheless, despite of the huge potential, many different results are available, both for salivary oral cancer diagnostics and for analysis of periodontal tissue. Accordingly, it is questionable if the reasons are methodological differences between the studies or maybe if the usage of miRNAs in general is unsuitable.

Therefore, this review article shows current results of in vitro and in vivo studies regarding periodontal diseases as well as results of selected current studies dealing with salivary miRNAs for oral cancer detection. The aim was to detect promising future perspectives, but also current challenges regarding this issue. In particular, methodological aspects are focused to detect potential limitations of miRNAs as noninvasive markers for periodontitis.

\section{Methods}

2.1. Search Strategy. Our literature research was performed using the online database PubMed (http://www.ncbi.nlm.nih .gov/pubmed). To obtain a broad overview of the available theme-specific publications, we used different keywords for our search. We used "miRNA AND periodontitis" as keywords to obtain results for periodontal diseases. We used "miRNA AND saliva AND oral disease" and "miRNA AND saliva AND cancer" as keywords to obtain relevant articles for noninvasive possibilities, that is, the use of saliva to investigate miRNAs in diseases of the oral cavity. Then, the reference lists of relevant articles were searched for further results. To interpret the results, relevant publications were searched and analyzed critically. Neither a meta-analysis nor other statistical comparisons of existing data were performed.

2.2. Selection Criteria. Only current full-length articles in English language were included. The results were verified for their relevance. The following selection criteria were defined: miRNA investigations for in vitro and in vivo studies examining periodontal disease. Additionally, in vivo human investigations regarding salivary miRNA analysis in oral cancer and precancer were included.
2.3. Selection Process. Following the literature search, all references were limited to relevant publications. In vivo periodontal disease investigations that did not address periodontal tissue were excluded. Salivary diagnostic studies that did not explicitly concentrate on miRNAs were excluded, and only investigations for oral cancer and precancer were included.

\section{Results}

\subsection{Periodontal Diseases}

3.1.1. In Vitro. Eight in vitro studies, which detected different miRNAs, were included for periodontal disease (Table 1). Nahid et al. (2011) examined the expression of cytokines associated with inflammation and differences in expression of cytokines and miRNA during infection with live and heatkilled bacteria in THP-1 monocytes. They demonstrated that expression of miR-146a is associated with infections caused by periodontal pathogenic bacteria in vitro [13]. Another study indicated that miR-146a is significantly overexpressed in THP-1 cells after stimulation with LPS from P.g. [14]. Furthermore, miR-146a was shown to be a negative regulator of TLR-NF $\kappa$ B pathway in human periodontal ligament cells after $P$. gingivalis LPS stimulation, therefore being involved in inflammatory response [15]. Moreover, expression of miR$146 \mathrm{a}$ and miR-146b-5p in human gingival fibroblasts after stimulation with P.g. LPS was also investigated in a study by Xie et al. (2013). They demonstrated that expression of these miRNAs significantly increased upon LPS stimulation and concluded that miR-146 could work as a negative regulator of inflammation in periodontal disease [16]. Differential expression of miR-146a and miR-155 in dental pulp and in gingival and periodontal fibroblasts after stimulation with LPS from Escherichia coli was also reported. The increased expression of miR-146a was the highest in gingival fibroblasts and decreased expression of miR-155 was only significant in gingival fibroblasts [17]. Naqvi et al. (2014) showed different expressions of miR-146a. In this in vitro study THP1 macrophages that were stimulated with LPS from Aggregatibacter actinomycetemcomitans, P.g., and also P.g. grown on cigarette smoke extract were examined. The different LPS caused both some identical and also varied expressions of miRNAs in human macrophages. Moreover, LPS from P.g. grown on cigarette smoke extract compared to LPS from unaffected P.g. caused differential miRNA expression, particularly that of miR-29b in human macrophages [18].

Results reported in other two studies were more heterogenous: Ouhara et al. (2014) demonstrated upregulated miR584 in human gingival epithelial cells after stimulation with P.g. [19]. Furthermore, Moffat and Lamont (2011) focused on miR-203 expression in gingival epithelial cells stimulated with P.g. In this study, 14 miRNAs displayed significant changes in expression after exposure to P.g.; of these miRNAs, miR-203 was examined in detail and showed higher expression in tissues after P.g. stimulation [20].

3.1.2. In Vivo Animal. Nahid et al. (2011) investigated expression of miR-146a, miR-132, and miR-155 in spleens and 


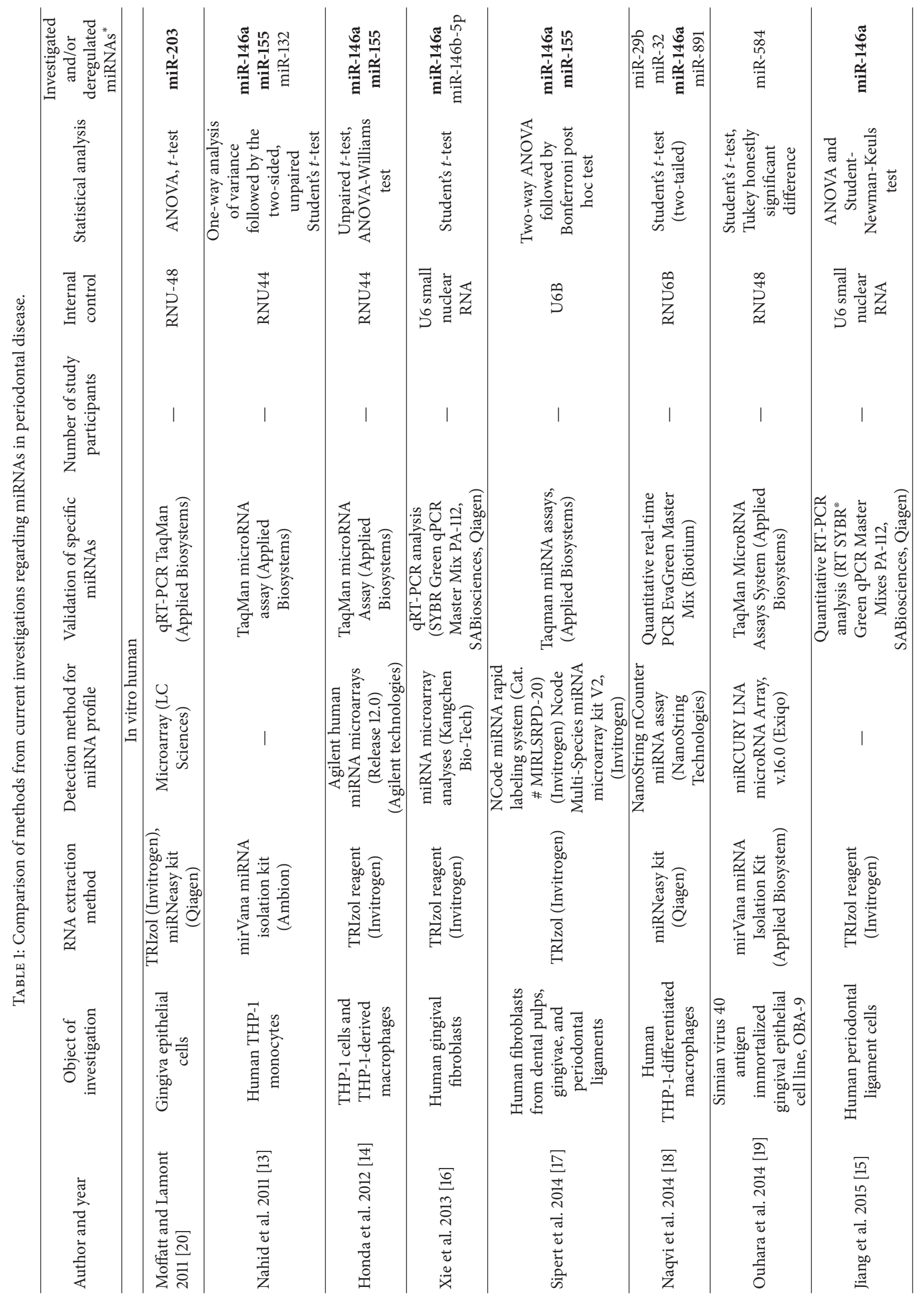




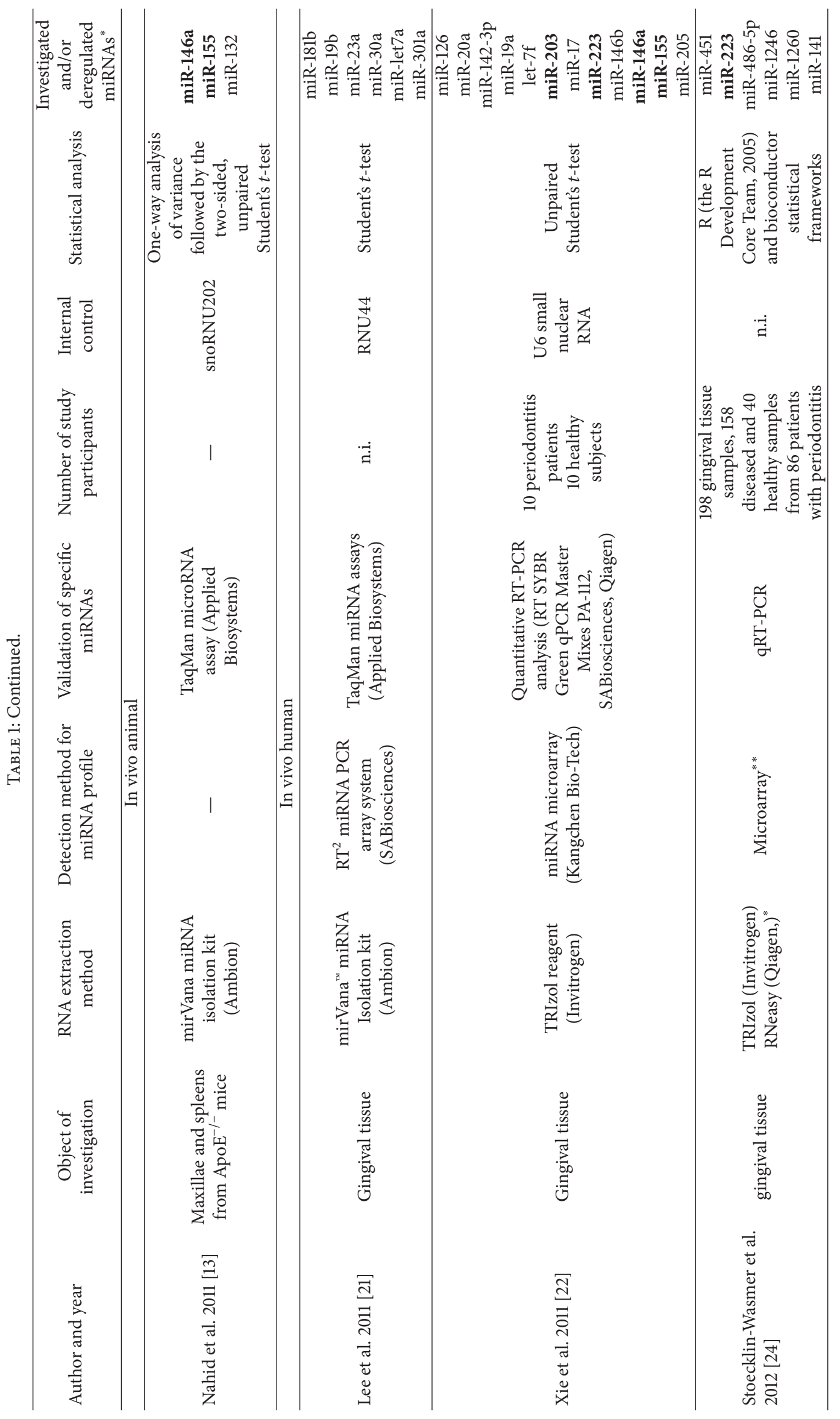




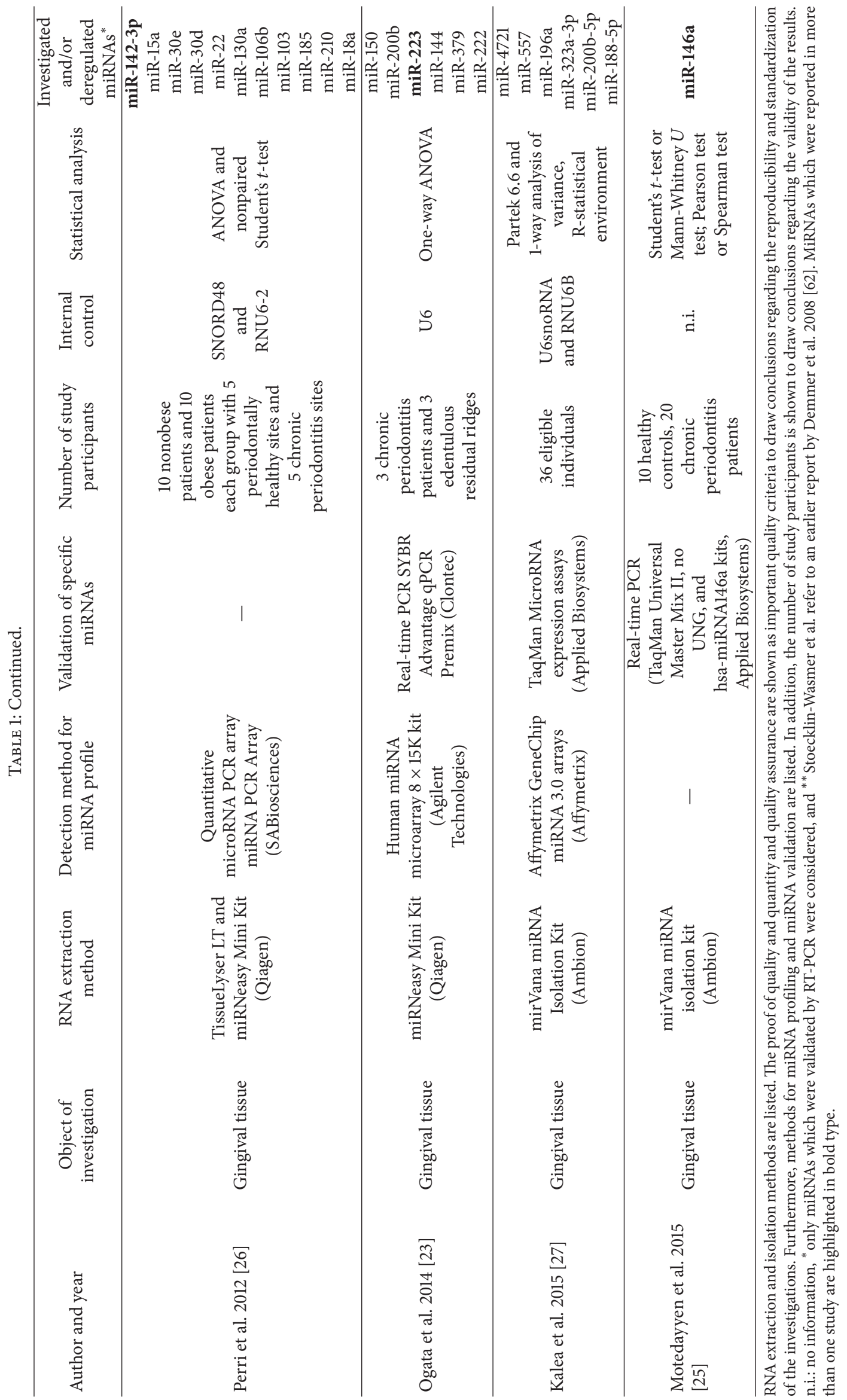


TABLE 2: Criteria for patient selection.

\begin{tabular}{|c|c|c|c|c|c|c|c|c|}
\hline \multirow{2}{*}{ Author and year } & \multicolumn{2}{|r|}{$\mathrm{PD}$} & \multicolumn{2}{|r|}{ CAL } & \multicolumn{2}{|c|}{$\mathrm{BOP}$} & \multicolumn{2}{|c|}{ Radiographic bone loss } \\
\hline & Healthy & Periodontitis & Healthy & Periodontitis & Healthy & Periodontitis & Healthy & Periodontitis \\
\hline Stoecklin-Wasmer et al. 2012 [24] & $\leq 4 \mathrm{~mm}$ & $>4 \mathrm{~mm}$ & $\leq 2 \mathrm{~mm}$ & $\geq 3 \mathrm{~mm}$ & negative & positive & n.i. & n.i. \\
\hline Xie et al. 2011 [22] & $<3 \mathrm{~mm}$ & $\geq 5 \mathrm{~mm}$ & $<1 \mathrm{~mm}$ & $\geq 3 \mathrm{~mm}$ & n.i. & * & No & Yes \\
\hline Lee et al. 2011 [21] & $\leq 3 \mathrm{~mm}$ & $>5 \mathrm{~mm}$ & none & $>3 \mathrm{~mm}$ & ** & n.i. & No & Yes \\
\hline Perri et al. 2012 [26] & $\leq 4 \mathrm{~mm}$ & $>5 \mathrm{~mm}$ & n.i. & n.i. & Negative & Positive & No & Yes \\
\hline Ogata et al. 2014 [23] & n.i. & $\geq 6 \mathrm{~mm}$ & n.i. & $>6 \mathrm{~mm}$ & n.i. & Positive & n.i. & n.i. \\
\hline Motedayyen et al. 2015 [25] & $<3 \mathrm{~mm}$ & n.i. & $<3 \mathrm{~mm}$ & n.i. & n.i. & n.i. & No & n.i. \\
\hline Kalea et al. 2015 [27] & n.i. & $>5 \mathrm{~mm}$ & n.i. & $* * *$ & n.i. & n.i. & n.i. & $* * *$ \\
\hline
\end{tabular}

PD: pocket depth; CAL: clinical attachment loss; BOP: bleeding on probing; n.i.: no information. ${ }^{*} \mathrm{GI}>1$; ${ }^{* *} \mathrm{BOP}$ in whole gingiva $<10 \%,{ }^{* * *}$ bone loss $>30 \%$.

maxillae of $\mathrm{ApoE}^{-/-}$mice after infection with periodontal pathogenic bacteria P.g., Treponema denticola and Tannerella forsythia. MiR-146a primarily showed a significant increase in both maxillae and spleens, while miR-132 and miR-155 showed only minor changes [13].

3.1.3. In Vivo Human. Seven in vivo studies investigating differential expression of miRNAs in human tissue samples were included (Table 1). In particular, Lee et al. (2011) compared the expression of inflammatory miRNAs in healthy and inflamed periodontal tissue. Microarray analysis indicated that expression of six miRNAs was upregulated more than eightfold and that expression of $22 \mathrm{miRNAs}$ increased more than fourfold in periodontal disease tissue compared with healthy tissue. Of these miRNAs, six could be validated by qRT-PCR [21]. A similar investigation considered miRNA profiles of healthy and inflamed gingival tissues. Microarray analysis of healthy and inflamed gingival tissues indicated that expression of five miRNAs was upregulated more than fivefold, 85 miRNAs, twofold to fivefold. Moreover, expression of 34 miRNAs was downregulated twofold to fivefold in inflamed tissue. 12 miRNAs were validated by qRT-PCR [22]. A study of Japanese patients identified 17 upregulated and 22 downregulated miRNAs; six were selected for validation [23]. A further investigation also detected differential miRNA expression by microarray analysis, wherein 91 upregulated and 68 downregulated miRNAs were found. Six miRNAs were validated, and potential target genes were investigated [24]. Furthermore, Motedayyen et al. (2015) showed a positive relationship between miR-146a and clinical parameters in patients with chronic periodontitis [25]. Thus, many miRNAs with differential expression as detected by microarray analysis have not been validated by qRT-PCR; these miRNAs will not be discussed (Table 1).

Additionally, two studies investigated the roles of miRNAs in periodontal disease in association with systemic factors and disease; thus, differential expression of miRNAs between obese periodontitis patients and nonobese periodontitis patients was examined. However, different, nonoverlapping miRNAs were reported in each investigation [26, 27].

Notably, criteria for patient selection and for classification of healthy and diseased patients were similar across all studies. However, differences exist between investigations in defining periodontal health and periodontitis (Table 2).
Additionally the number of study participants for in vivo investigations varied enormously between studies with ten patients or less for each group in most cases (Table 1). Likewise, methodological approach was similar across studies: microarray based technology was often used to screen and identify miRNAs with differential expression, and qRT-PCR was used for subsequent validation of top-hits. However, detailed methods were different; thus, for example, different miRNA isolation methods were used. Also differences in statistical analysis are conspicuous, especially internal controls that distinguish between the studies (Table 1).

\subsection{MiRNA Detection in Saliva as a Noninvasive Diagnostic} Tool. Nine investigations were included that illustrate the potential of saliva as noninvasive diagnostic tool for miRNA analyzes. We focused on salivary miRNA species analysis as a diagnostic method for oral cancer and precancer detection, because no results of appropriate dental and periodontal disease investigations could be found. Six of included studies examined oral squamous cell carcinoma, one precancerous lesions, one esophageal cancer, and one tumor of parotid gland. Park et al. (2009) showed presence of miRNA species in whole saliva and in saliva supernatants and described differential expression of miR-125a and miR-200a between cases and controls. Besides that stability of exogenous (miR124a) and endogenous (miR-191) miRNA in saliva was also examined in this study, and endogenous miRNA was shown to have higher stability than exogenous miRNA [28]. Another investigation found that miR-9, miR-191, and miR-134 appeared to be potential markers for noninvasive diagnosis of oral squamous cell carcinoma using saliva. Additionally, this study describes a reliable isolation method for miRNAs from even small volumes of saliva [29]. In a study by Hung et al. (2016) salivary miRNAs, specifically miR-31 and miR-21 were investigated in oral premalignant disorders, particularly showing miR-31 to be a sufficient marker for high risk disorders and malignant transformation [30]. Also Liu et al. (2012) examined miR-31, and its expression in saliva was compared with its expression in plasma; thereby, a correlation could be shown. These authors also observed a decrease in expression of miR-31 after surgical resection of primary tumor [31]. Similarly, Duz et al. (2016) reduced miR-139-5p expression in patients with tongue carcinoma and normalization after surgical treatment [32]. 
In a study investigating expression of salivary miRNAs in premalignant lesions of oral cavity, a correlation was found between deregulated miRNA expression in tissue and saliva, although miRNA concentrations in saliva were lower [33]. A further investigation compared expression of miRNA in saliva between patients with oral squamous cell carcinoma, patients with oral squamous cell carcinoma in remission, patients with oral lichen planus, and a healthy control group. In addition to differences in miRNA expression between the groups, miR-27b was significantly overexpressed in saliva of patients with oral squamous cell carcinoma. Furthermore, completely different profiles of miRNAs could be found in cancerous tissues compared to biological fluids [34]. Additionally, another study found miRNA-184 to have the potential to distinguish between OSCC and potentially malignant disorders [35].

In addition, altered miRNA expression in saliva was also verified for esophageal cancer [36] and parotid gland tumors [37]. Their methodologies are shown in Table 3. In principle, methods of measurement (microarray, qRT-PCR) are similar between the studies. Remarkable differences could be found regarding form of saliva, extraction methods, statistical analysis, and internal controls. The number of study participants was already higher than for periodontitis. However, heterogeneous groups sizes with partly seven or eight patients each group $[33,34]$ and partly 50 patients and more each group $[28,29]$ were investigated (Table 3 ).

\section{Discussion}

Several studies investigating putative role of miRNAs in periodontal diseases have been performed; however, spectrum of miRNAs identified was substantially heterogeneous. Both in vitro and in vivo investigations were performed for periodontal disease, and five miRNAs, that is, miR-142-3p, miR-146a, miR-155, miR-203, and miR-223, were validated in more than one study. Accordingly, we can conclude that these miRNAs may play important role and thus could become potential markers for periodontal disease. However, majority of identified miRNA species differed significantly across studies (Table 1).

MiR-146a and miR-155 were dominant in in vitro studies. Otherwise, these miRNAs have also been identified in other inflammatory diseases and various cancers. MiR-146a seems to play a role in multifarious diseases and its function in bacterial infections has already been discussed [38]. MiR-155 has also been mentioned [39]. LPS may have a substantial effect on the expression of miR-146a and miR-155 [40]. Consequently, their altered expression during periodontal disease is not surprising because bacterial virulence factors such as LPS are relevant in pathogenesis of periodontitis [41]. These miRNAs are associated with many other inflammatory diseases, which might be indicative of a limited specificity. Additionally, miR-142-3p is associated with inflammation and LPS exposure [42, 43]. MiR-203 may also play an important role in inflammation and correlate with LPS exposure [44]. Interestingly, miR-203 expression is associated with immune reaction of skin $[45,46]$. MiR-203 expression in keratinocytes is important; therefore, this miRNA may also be involved in gingivitis and periodontitis as determined by its effect on gingival keratinocytes. Finally, miR-223 also plays an important role in inflammation and LPS exposure [47]. Accordingly, correlations between these five miRNAs and periodontal disease are possible. Interestingly, these miRNAs appear to play a role in oral cancer. Thus, their roles in periodontal disease should be further examined (Table 4) as it might indicate a link between periodontitis and head and neck squamous cell carcinoma (HNSCC). Figure 1 shows a summary of the interactions between periodontitis and HNSCC [48], and some potentially involved miRNAs. In this context, miR-29b, which showed an in vitro correlation with LPS from P.g. grown on cigarette smoke extract [18], has also been examined for oral cancer [49]. These findings raise the question if these miRNAs could serve as diagnostic markers for periodontal diseases or simply reflect an increased inflammatory state associated with various diseases.

Another problem is the great diversity of results. Although many miRNAs were found, convergence between the investigations, particularly in vivo, is small, resulting in conflicting conclusions. Reasons for differences between investigations of periodontal disease may differ. On the one hand, small variety in patient selection and different clinical procedures potentially causes these differences (Table 2). For future studies an evident graduation, for example, Page and Eke 2007, should be used for standardization [50]. On the other hand, variations in methods used for miRNA detection could play a crucial role (Table 3). A comparison of methods used suggests that miRNA detection procedures were quite similar. However, many differences in detailed procedures are evident. Using microarray and quantitative RT-PCR for analyzing and validating miRNAs are essential components of most investigations. However, detailed procedures for methods primarily differ, so several arrays from different manufacturing companies were performed. Importantly, differences in all of chosen criteria can be found between the investigations. Besides variations in statistical analyses it is important that internal controls differed in most cases.

Another aspect that could be crucial for the varying results is the small number of study participants. One study even investigated only three healthy and three diseased individuals [23]. A large group of 86 patients was selected only once [24]. The use of small sample sizes increases vulnerability to a range of errors and biases and may be a major reason for heterogeneous study results and potential false-positive findings. Similar issues were faced in early human genetic association studies, where many of reported genotype-phenotype associations could not be replicated in subsequent studies [51, 52]. Today, replication of initial findings in second cohort is considered essential for establishing the credibility of a genotype-phenotype association [53] and similar approaches could be adopted for studies reporting associations between miRNAs and diseases. Clearly, complex reason for differences in results exists. Different methods, clinical criteria, small groups of patients, and small specificity of miRNAs for periodontal disease could be causal complex.

In summary, comparability of results for the roles of miRNAs in periodontal disease is questionable. Potential 


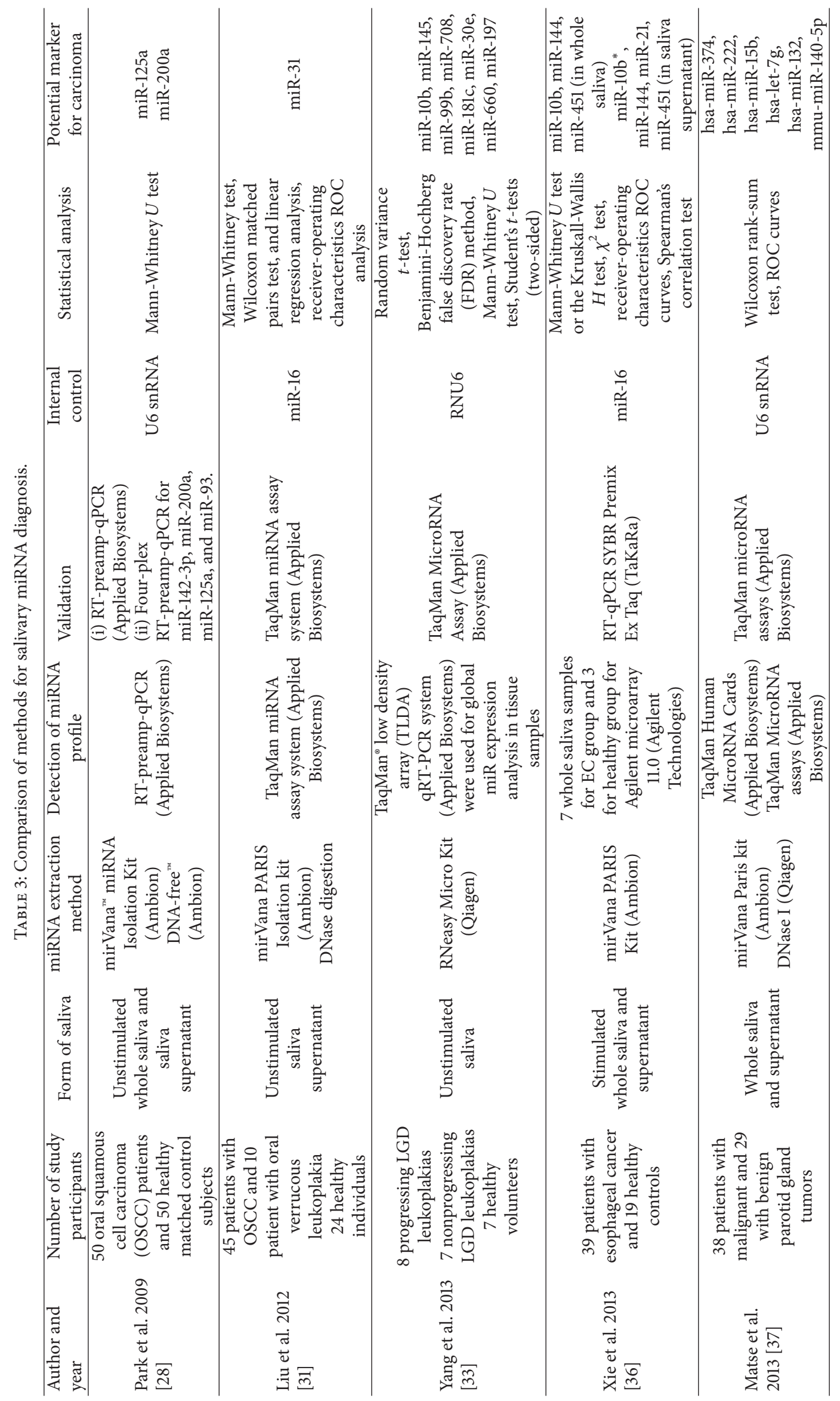




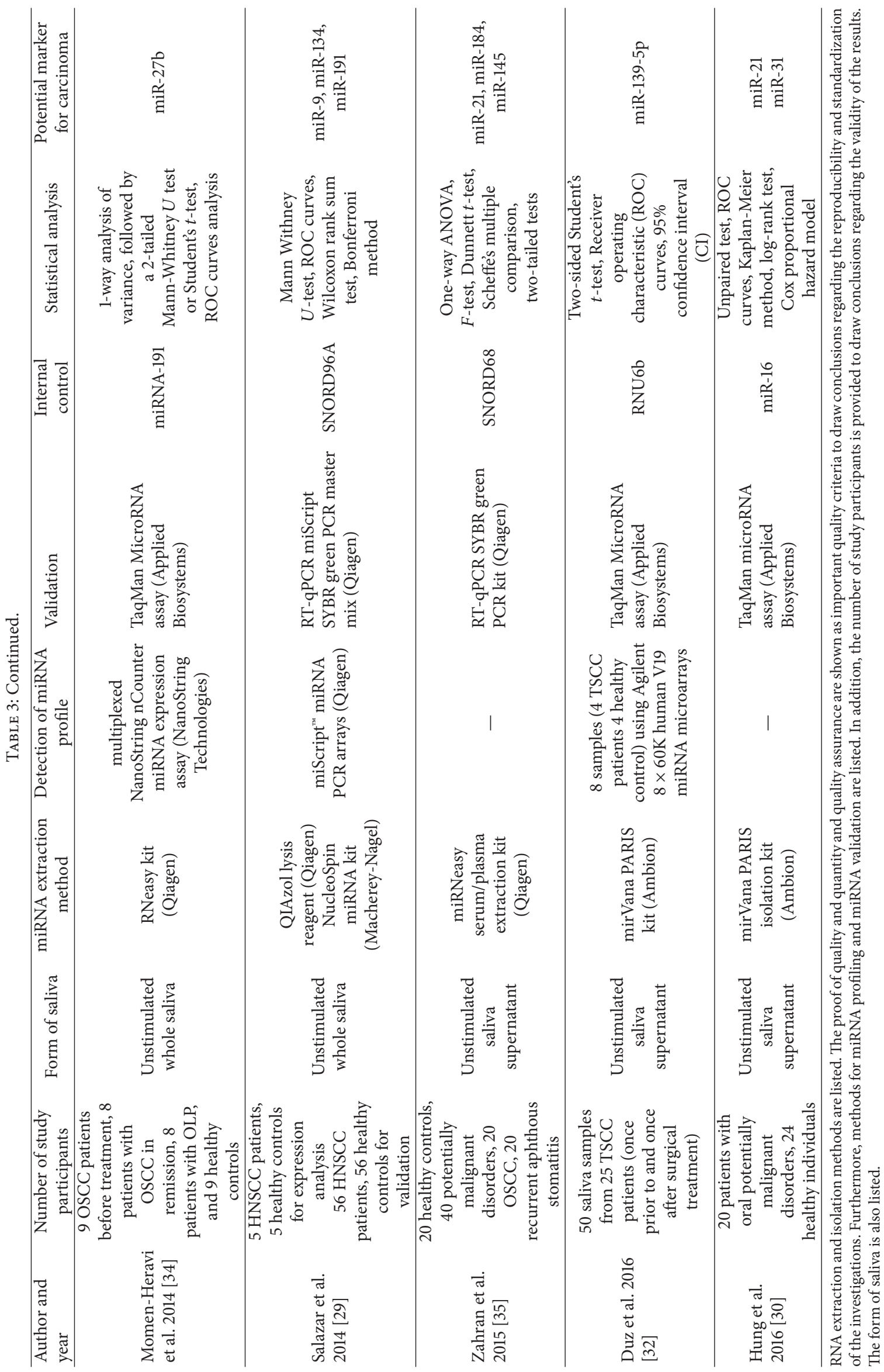


TABLE 4: Comparison of miRNas frequently mentioned in connection with periodontal disease with results from selected oral cancer and precancer investigations.

\begin{tabular}{|c|c|c|c|c|c|c|}
\hline & $\begin{array}{c}\text { Kozaki et al. } \\
2008 \text { [63] }\end{array}$ & $\begin{array}{l}\text { Park et al. } \\
2009 \text { [28] }\end{array}$ & $\begin{array}{c}\text { Cervigne et al. } \\
2009 \text { [64] }\end{array}$ & $\begin{array}{c}\text { Scapoli et al. } \\
2010 \text { [65] }\end{array}$ & $\begin{array}{c}\text { Lajer et al. } \\
2011 \text { [66] }\end{array}$ & $\begin{array}{c}\text { Lundegard et al. } \\
2015 \text { [56] }\end{array}$ \\
\hline miR-146a & $\mathrm{X}$ & & $\mathrm{X}$ & $\mathrm{X}$ & $\mathrm{X}$ & \\
\hline miR-155 & & & $\mathrm{X}$ & $\mathrm{X}$ & $\mathrm{X}$ & \\
\hline miR-203 & $\mathrm{X}$ & & & & & $\mathrm{X}$ \\
\hline miR-142-3p & & $\mathrm{X}$ & $\mathrm{X}$ & & & \\
\hline miR-223 & & & $\mathrm{X}$ & & $\mathrm{X}$ & \\
\hline
\end{tabular}

Each miRNA that was validated in the context of periodontal disease was also mentioned in investigations for cancer and precancer of the oral cavity. This table provides a small exemplary overview. Consequently, even more studies with similar results are not shown here.

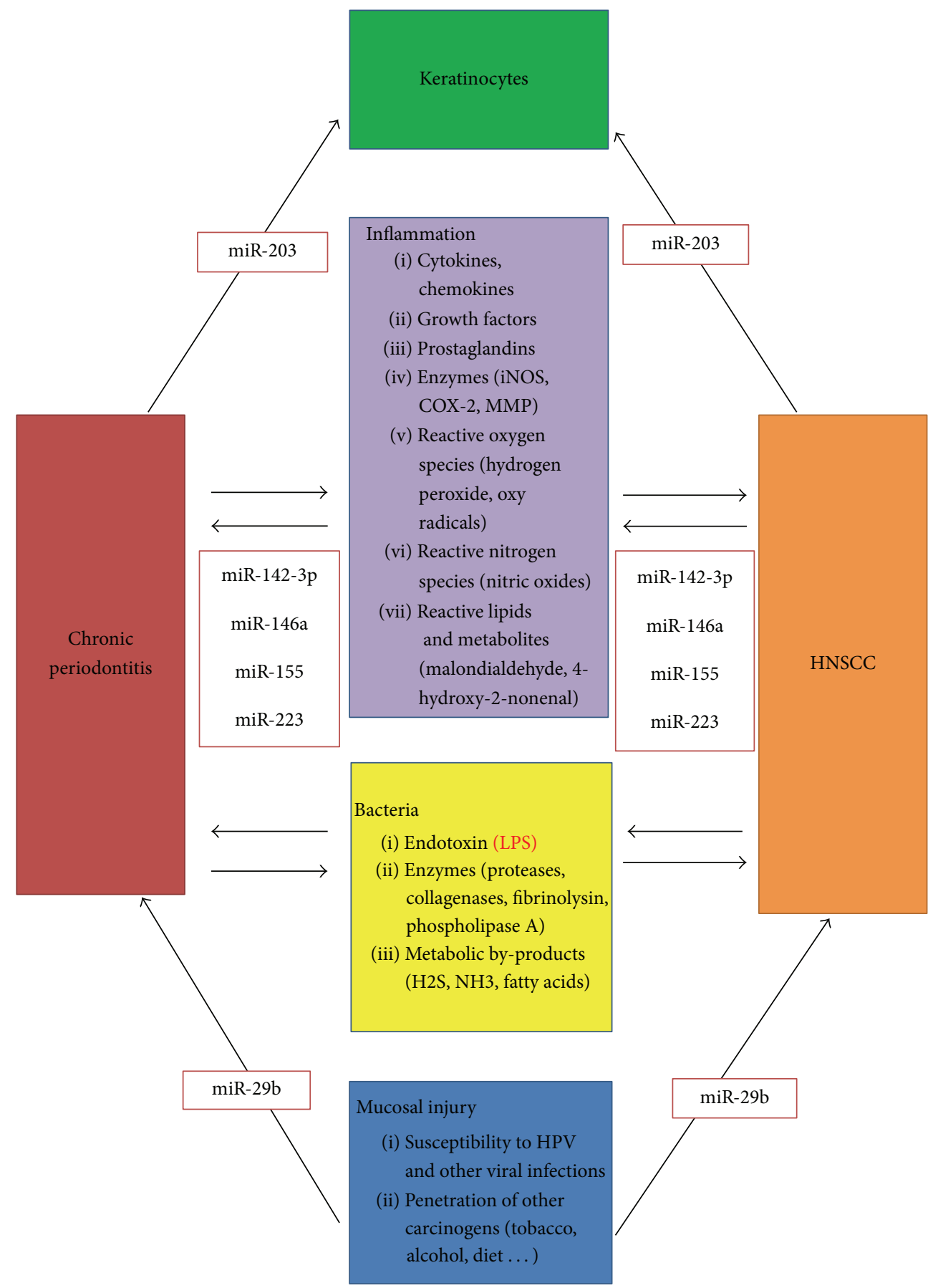

FIGURE 1: Model for the relationship among chronic periodontitis, HNSCC (modif. Han et al. 2014 [48]) and potential miRNAs that may be involved in the corresponding processes. 
candidate miRNAs as promising markers of periodontal disease do not appear to be specific for periodontitis.

Use of salivary miRNAs as noninvasive diagnostic markers has been studied in context of oral cancer, precancer, esophageal cancer, and parotid gland cancer so far. Again, reported results of these studies were quite divergent with little overlap in identified miRNA species. Importantly, these investigations used similar methods for miRNA detection in periodontal tissue and saliva; however, detailed methods are quite different (Tables 1 and 3 ).

In principle, detectability of miRNAs in saliva seems certainly possible, so, for example, a current study was able to detect noncoding RNAs in saliva: 127 to 418 miRNAs could be detected in each sample of human cell free saliva, with miR-223-3p being most abundant [54]. Interestingly, miR223 is also found in periodontal tissue. Different studies have examined saliva to identify miRNAs as potential markers for oral cancer and precancer and report remarkably diverse results (Table 3). Strangely, basic principles of investigations were similar, but different internal controls, methods for miRNA isolation, statistical analysis, and forms of saliva (stimulated/unstimulated, whole saliva/supernatant) were chosen. Notably, number of study participants is already higher in these investigations than in studies of periodontitis, but also heterogeneous with partly small group sizes (Tables 1 and 3). In addition, form of saliva may play an important role, especially whether stimulated or unstimulated saliva is used may be relevant as well as exact procedure for obtaining saliva and criteria for patient selection. Furthermore, lack of stability of exogenous miRNAs in saliva may result in quick changes of miRNA concentrations from bacteria and inflammatory reactions [28]. Potential concentrations of miRNAs in body fluids in exosomes could also affect their detectability in human saliva samples [55]. Beside of that, however, it was mentioned that there are vesicle-free noncoding RNAs in saliva [54]. Furthermore, it is questionable whether identified miRNAs from tissue investigations are also potential markers for salivary diagnostic methods. The finding of completely different miRNA expression between cancerous tissue and body fluids [34] could be similar for periodontitis.

Detailed procedures of miRNA extraction and detection could also be relevant. In a recent study by Lundegard et al. (2015) expression of miR-203 was examined in whole saliva using two different PCR methods. The study concluded that detecting low levels of miRNA in saliva is difficult; more efficient extraction methods and more sensitive PCR techniques are necessary to use saliva as a reproducible source of miRNAs [56]. Because of differences in methods used, it is questionable if investigations are comparable at all. Some miRNAs may have variable expression between different microarray, PCR, and preparation methods [57]. Reproducibility and standardization of procedure appear relevant. Validated protocols such as those already described [58] may help to standardize the procedures. This would allow better reproducibility and provide more meaningful and more comparable results. This is supported by results of Hung et al. and Liu et al. both showing miR-31 to be a potential marker for malignant tumor [30,31]. As shown in Table 3, both studies used same form of saliva, extraction, and validation methods as well as internal controls (Table 3). Accordingly, use of saliva for diagnosis of periodontal disease under condition of uniform methods seems conceivable.

However, the mechanism of miRNA infiltration in saliva during periodontal diseases must be considered. Thereby, exosomes might play a key role in miRNA transport from different cells into saliva [59]. In periodontal tissue, the junctional epithelial layers might be of highest relevance in this context. The junctional epithelium contains only few desmosomes and has therefore widened intercellular spaces [60]. Accordingly, this epithelium allows transport of several molecules between tissue and gingival crevicular fluid (GCF) [61]. Additionally, an enlarged permeability with an increase of GCF flow is observed during gingival and periodontal inflammation [61]. Consequently, high amounts of miRNA might pass the junctional epithelium, arriving GCF and thus saliva.

If comparable results can be achieved, further investigations will be of interest. For example, changes in miRNA expression after surgical resection of a tumor, as already described $[3,32]$, could become clinically useful by demonstrating improved periodontal conditions after therapy as well. Therefore, it could be assessed whether periodontitis therapy was successful by analyzing expression of specific miRNAs. Additionally, correlation between periodontitis and systemic diseases could be illustrated by altered miRNA expression [26, 27]. Although this topic seems to have great potential to provide further insights regarding oral disease, a critical view is needed. Currently, we have insufficient knowledge regarding oral disease and the roles of miRNAs in pathological processes. At present it is impossible to provide a clear statement regarding real relevance and possibilities of miRNA analysis. Nevertheless, using miRNAs to understand oral diseases, particularly periodontitis, and potential use of miRNAs as noninvasive markers or as therapeutic targets could be a great approach, which justifies basic research in any case. In conclusion, we can confirm unequivocally that salivary miRNA diagnosis for periodontal disease is a revolutionary idea. However, considering that additional investigations and standardized methods are required, possibilities of exploiting potential could be estimated only in the future.

\section{Conclusion}

Besides similar methods regarding miRNA extraction, profiling, and validation, there are methodical differences between studies, especially in internal controls and sample size, resulting in heterogeneous results. In principle, salivary miRNA diagnostic methods seem feasible. However, in our opinion, standardized criteria and protocols should be established and followed exactly to obtain comparable results. Five miRNAs related to inflammation are available, which may be used as potential markers for periodontitis. However, their detectability and expression in saliva and, accordingly, their importance as noninvasive markers are questionable.

\section{Competing Interests}

The authors declare that they have no conflict of interests. 


\section{Acknowledgments}

The authors acknowledge support from the German Research Foundation (DFG) and Universität Leipzig within the program of Open Access Publishing.

\section{References}

[1] D. P. Bartel, "MicroRNAs: genomics, biogenesis, mechanism, and function," Cell, vol. 116, no. 2, pp. 281-297, 2004.

[2] R. M. O'Connell, D. S. Rao, A. A. Chaudhuri, and D. Baltimore, "Physiological and pathological roles for microRNAs in the immune system," Nature Reviews Immunology, vol. 10, no. 2, pp. 111-122, 2010.

[3] J. Hao, S. Zhao, Y. Zhang et al., "Emerging role of MicroRNAs in cancer and cancer stem cells," Journal of Cellular Biochemistry, vol. 115, no. 4, pp. 605-610, 2014.

[4] S. Qin and C. Zhang, "MicroRNAs in vascular disease," Journal of Cardiovascular Pharmacology, vol. 57, no. 1, pp. 8-12, 2011.

[5] R. Maqbool and M. U. Hussain, "MicroRNAs and human diseases: diagnostic and therapeutic potential," Cell and Tissue Research, vol. 358, no. 1, pp. 1-15, 2014.

[6] D. Chen, R. J. Cabay, Y. Jin et al., "MicroRNA deregulations in head and neck squamous cell carcinomas," Journal of Oral \& Maxillofacial Research, vol. 4, no. 1, article e2, 2013.

[7] R. Roy, N. De Sarkar, S. Ghose et al., "Association between risk of oral precancer and genetic variations in microRNA and related processing genes," Journal of Biomedical Science, vol. 21, article $48,2014$.

[8] C. Staedel and F. Darfeuille, "MicroRNAs and bacterial infection," Cellular Microbiology, vol. 15, no. 9, pp. 1496-1507, 2013.

[9] S. H. Kim, S. Y. Lee, Y. M. Lee, and Y. K. Lee, "MicroRNAs as biomarkers for dental diseases," Singapore Dental Journal, vol. 36, pp. 18-22, 2015.

[10] M. Kebschull and P. N. Papapanou, "Mini but mighty: microRNAs in the pathobiology of periodontal disease," Periodontology 2000, vol. 69, no. 1, pp. 201-220, 2015.

[11] R. S. Patel, A. Jakymiw, B. Yao et al., "High resolution of microRNA signatures in human whole saliva," Archives of Oral Biology, vol. 56, no. 12, pp. 1506-1513, 2011.

[12] X. Lin, H.-C. Lo, D. T. W. Wong, and X. Xiao, "Noncoding RNAs in human saliva as potential disease biomarkers," Frontiers in Genetics, vol. 6, article 175, 2015.

[13] M. A. Nahid, M. Rivera, A. Lucas, E. K. L. Chan, and L. Kesavalu, "Polymicrobial infection with periodontal pathogens specifically enhances microRNA miR-146a in ApoE-/- mice during experimental periodontal disease," Infection and Immunity, vol. 79, no. 4, pp. 1597-1605, 2011.

[14] T. Honda, N. Takahashi, S. Miyauchi, and K. Yamazaki, "Porphyromonas gingivalis lipopolysaccharide induces miR-146a without altering the production of inflammatory cytokines," Biochemical and Biophysical Research Communications, vol. 420, no. 4, pp. 918-925, 2012.

[15] S.-Y. Jiang, D. Xue, Y.-F. Xie et al., "The negative feedback regulation of microRNA-146a in human periodontal ligament cells after Porphyromonas gingivalis lipopolysaccharide stimulation," Inflammation Research, vol. 64, no. 6, pp. 441-451, 2015.

[16] Y. F. Xie, R. Shu, S. Y. Jiang, D. L. Liu, J. Ni, and X. L. Zhang, "MicroRNA-146 inhibits pro-inflammatory cytokine secretion through IL-1 receptor-associated kinase 1 in human gingival fibroblasts," Journal of Inflammation, vol. 10, article 20, 2013.

[17] C. R. Sipert, A. C. Morandini, T. J. Dionísio, A. J. Trachtenberg, W. P. Kuo, and C. F. Santos, "MicroRNA-146a and microRNA155 show tissue-dependent expression in dental pulp, gingival and periodontal ligament fibroblasts in vitro," Journal of Oral Science, vol. 56, no. 2, pp. 157-164, 2014.

[18] A. R. Naqvi, J. B. Fordham, A. Khan, and S. Nares, "MicroRNAs responsive to Aggregatibacter actinomycetemcomitans and Porphyromonas gingivalis LPS modulate expression of genes regulating innate immunity in human macrophages," Innate Immunity, vol. 20, no. 5, pp. 540-551, 2014.

[19] K. Ouhara, I. J. Savitri, T. Fujita et al., "MiR-584 expressed in human gingival epithelial cells is induced by porphyromonas gingivalis stimulation and regulates interleukin- 8 production via lactoferrin receptor," Journal of Periodontology, vol. 85, no. 6, pp. e198-e204, 2014.

[20] C. E. Moffatt and R. J. Lamont, "Porphyromonas gingivalis induction of microRNA-203 expression controls suppressor of cytokine signaling 3 in gingival epithelial cells," Infection and Immunity, vol. 79, no. 7, pp. 2632-2637, 2011.

[21] Y. H. Lee, H. S. Na, S. Y. Jeong, S. H. Jeong, H. R. Park, and J. Chung, "Comparison of inflammatory microRNA expression in healthy and periodontitis tissues," Biocell, vol. 35, no. 2, pp. 43-49, 2011.

[22] Y. F. Xie, R. Shu, S. Y. Jiang, D. L. Liu, and X. L. Zhang, "Comparison of microRNA profiles of human periodontal diseased and healthy gingival tissues," International Journal of Oral Science, vol. 3, no. 3, pp. 125-134, 2011.

[23] Y. Ogata, S. Matsui, A. Kato, L. Zhou, Y. Nakayama, and H. Taka, "MicroRNA expression in inflamed and noninflamed gingival tissues from Japanese patients," Journal of Oral Science, vol. 56, no. 4, pp. 253-260, 2014.

[24] C. Stoecklin-Wasmer, P. Guarnieri, R. Celenti, R. T. Demmer, M. Kebschull, and P. N. Papapanou, "MicroRNAs and their target genes in gingival tissues," Journal of Dental Research, vol. 91, no. 10, pp. 934-940, 2012.

[25] H. Motedayyen, S. Ghotloo, M. Saffari, M. Sattari, and R. Amid, "Evaluation of MicroRNA-146a and its targets in gingival tissues of patients with chronic periodontitis," Journal of Periodontology, vol. 86, no. 12, pp. 1380-1385, 2015.

[26] R. Perri, S. Nares, S. Zhang, S. P. Barros, and S. Offenbacher, "MicroRNA modulation in obesity and periodontitis," Journal of Dental Research, vol. 91, no. 1, pp. 33-38, 2012.

[27] A. Z. Kalea, R. Hoteit, J. Suvan et al., "Upregulation of gingival tissue miR-200b in obese periodontitis subjects," Journal of Dental Research, vol. 94, pp. 59-69, 2015.

[28] N. J. Park, H. Zhou, D. Elashoff et al., "Salivary microRNA: discovery, characterization, and clinical utility for oral cancer detection," Clinical Cancer Research, vol. 15, no. 17, pp. 54735477, 2009.

[29] C. Salazar, R. Nagadia, P. Pandit et al., "A novel saliva-based microRNA biomarker panel to detect head and neck cancers," Cellular Oncology, vol. 37, no. 5, pp. 331-338, 2014.

[30] K.-F. Hung, C.-J. Liu, P.-C. Chiu et al., "MicroRNA-31 upregulation predicts increased risk of progression of oral potentially malignant disorder," Oral Oncology, vol. 53, pp. 42-47, 2016.

[31] C.-J. Liu, S.-C. Lin, C.-C. Yang, H.-W. Cheng, and K.-W. Chang, "Exploiting salivary miR-31 as a clinical biomarker of oral squamous cell carcinoma," Head and Neck, vol. 34, no. 2, pp. 219-224, 2012. 
[32] M. B. Duz, O. F. Karatas, E. Guzel et al., "Identification of miR-139-5p as a saliva biomarker for tongue squamous cell carcinoma: A Pilot Study," Cellular Oncology, vol. 39, no. 2, pp. 187-193, 2016.

[33] Y. Yang, Y. X. Li, X. Yang, L. Jiang, Z. J. Zhou, and Y. Q. Zhu, "Progress risk assessment of oral premalignant lesions with saliva miRNA analysis," BMC Cancer, vol. 13, article 129, 2013.

[34] F. Momen-Heravi, A. J. Trachtenberg, W. P. Kuo, and Y. S. Cheng, "Genomewide study of salivary MicroRNAs for detection of oral cancer," Journal of Dental Research, vol. 93, supplement 7, pp. 86S-93S, 2014.

[35] F. Zahran, D. Ghalwash, O. Shaker, K. Al-Johani, and C. Scully, "Salivary microRNAs in oral cancer," Oral Diseases, vol. 21, no. 6, pp. 739-747, 2015.

[36] Z. Xie, G. Chen, X. Zhang et al., "Salivary microRNAs as promising biomarkers for detection of esophageal cancer," PLoS ONE, vol. 8, no. 4, Article ID e57502, 2013.

[37] J. H. Matse, J. Yoshizawa, X. Wang et al., "Discovery and prevalidation of salivary extracellular microRNA biomarkers panel for the noninvasive detection of benign and malignant parotid gland tumors," Clinical Cancer Research, vol. 19, no. 11, pp. 30323038, 2013.

[38] Z. Liu, B. Xiao, B. Tang et al., "Up-regulated microRNA-146a negatively modulate Helicobacter pylori-induced inflammatory response in human gastric epithelial cells," Microbes and Infection, vol. 12, no. 11, pp. 854-863, 2010.

[39] L. N. Schulte, A. J. Westermann, and J. Vogel, "Differential activation and functional specialization of miR-146 and miR155 in innate immune sensing," Nucleic Acids Research, vol. 41, no. 1, pp. 542-553, 2013.

[40] Y. Cheng, W. Kuang, Y. Hao et al., "Downregulation of miR-27a* and miR-532-5p and upregulation of mir-146a and mir-155 in LPS-induced RAW264.7 Macrophage cells," Inflammation, vol. 35, no. 4, pp. 1308-1313, 2012.

[41] D. Mayrand and S. C. Holt, "Biology of asaccharolytic blackpigmented Bacteroides species," Microbiological Reviews, vol. 52, no. 1, pp. 134-152, 1988.

[42] S. A. Moschos, A. E. Williams, M. M. Perry, M. A. Birrell, M. G. Belvisi, and M. A. Lindsay, "Expression profiling in vivo demonstrates rapid changes in lung microRNA levels following lipopolysaccharide-induced inflammation but not in the antiinflammatory action of glucocorticoids," BMC Genomics, vol. 8, article 240, 2007.

[43] G. Xu, Z. Zhang, J. Wei et al., "MicroR-142-3p down-regulates IRAK-1 in response to Mycobacterium bovis BCG infection in macrophages," Tuberculosis, vol. 93, no. 6, pp. 606-611, 2013.

[44] X.-F. Ke, J. Fang, X.-N. Wu, and C.-H. Yu, "MicroRNA-203 accelerates apoptosis in LPS-stimulated alveolar epithelial cells by targeting PIK3CA," Biochemical and Biophysical Research Communications, vol. 450, no. 4, pp. 1297-1303, 2014.

[45] M. N. Primo, R. O. Bak, B. Schibler, and J. G. Mikkelsen, "Regulation of pro-inflammatory cytokines TNF $\alpha$ and IL24 by microRNA-203 in primary keratinocytes," Cytokine, vol. 60, no. 3, pp. 741-748, 2012.

[46] T. Wei, N. Xu, F. Meisgen, M. Ståhle, E. Sonkoly, and A. Pivarcsi, "Interleukin-8 is regulated by miR-203 at the posttranscriptional level in primary human keratinocytes," European Journal of Dermatology, 2013.

[47] M. Haneklaus, M. Gerlic, L. A. J. O’Neill, and S. L. Masters, "MiR-223: infection, inflammation and cancer," Journal of Internal Medicine, vol. 274, no. 3, pp. 215-226, 2013.
[48] Y. W. Han, W. Houcken, B. G. Loos, H. A. Schenkein, and M. Tezal, "Periodontal disease, atherosclerosis, adverse pregnancy outcomes, and head-and-neck cancer," Advances in Dental Research, vol. 26, no. 1, pp. 47-55, 2014.

[49] C. N. Yang, Y. T. Deng, J. Y. Tang et al., "MicroRNA-29b regulates migration in oral squamous cell carcinoma and its clinical significance," Oral Oncology, vol. 51, no. 2, pp. 170-177, 2016.

[50] R. C. Page and P. I. Eke, "Case definitions for use in populationbased surveillance of periodontitis," Journal of Periodontology, vol. 78, no. 7, pp. 1387-1399, 2007.

[51] J. N. Hirschhorn, K. Lohmueller, E. Byrne, and K. Hirschhorn, "A comprehensive review of genetic association studies," Genetics in Medicine, vol. 4, no. 2, pp. 45-61, 2002.

[52] A. T. Hattersley and M. I. McCarthy, "What makes a good genetic association study?" The Lancet, vol. 366, no. 9493, pp. 1315-1323, 2005.

[53] S. J. Chanock, T. Manolio, M. Boehnke et al., "Replicating genotype-phenotype associations," Nature, vol. 447, no. 7145, pp. 655-660, 2007.

[54] J. H. Bahn, Q. Zhang, F. Li et al., "The landscape of microRNA, piwi-interacting RNA, and circular RNA in human saliva," Clinical Chemistry, vol. 61, no. 1, pp. 221-230, 2015.

[55] A. Gallo, M. Tandon, I. Alevizos, and G. G. Illei, “The majority of microRNAs detectable in serum and saliva is concentrated in exosomes," PLoS ONE, vol. 7, no. 3, article e30679, 2012.

[56] M. Lundegard, K. Nylander, and K. Danielsson, "Difficulties detecting miRNA-203 in human whole saliva by the use of PCR," Medicina Oral, Patologia Oral y Cirugia Bucal, vol. 20, no. 2, pp. e130-e134, 2015.

[57] R. A. Ach, H. Wang, and B. Curry, "Measuring microRNAs: comparisons of microarray and quantitative PCR measurements, and of different total RNA prep methods," BMC Biotechnology, vol. 8, article 69, 2008.

[58] J. M. Yoshizawa and D. T. W. Wong, "Salivary microRNAs and oral cancer detection," Methods in Molecular Biology, vol. 936, pp. 313-324, 2013.

[59] V. Palanisamy, S. Sharma, A. Deshpande, H. Zhou, J. Gimzewski, and D. T. Wong, "Nanostructural and transcriptomic analyses of human saliva derived exosomes," PLoS ONE, vol. 5, article e8577, 2010.

[60] S. Hatakeyama, T. Yaegashi, Y. Oikawa et al., "Expression pattern of adhesion molecules in junctional epithelium differs from that in other gingival epithelia," Journal of Periodontal Research, vol. 41, no. 4, pp. 322-328, 2006.

[61] D. D. Bosshardt and N. P. Lang, "The junctional epithelium: from health to disease," Journal of Dental Research, vol. 84, no. 1, pp. 9-20, 2005.

[62] R. T. Demmer, J. H. Behle, D. L. Wolf et al., "Transcriptomes in healthy and diseased gingival tissues," Journal of Periodontology, vol. 79, no. 11, pp. 2112-2124, 2008.

[63] K. Kozaki, I. Imoto, S. Mogi, K. Omura, and J. Inazawa, "Exploration of tumor-suppressive microRNAs silenced by DNA hypermethylation in oral cancer," Cancer Research, vol. 68, no. 7, pp. 2094-2105, 2008.

[64] N. K. Cervigne, P. P. Reis, J. Machado et al., "Identification of a microRNA signature associated with progression of leukoplakia to oral carcinoma," Human Molecular Genetics, vol. 18, no. 24, pp. 4818-4829, 2009.

[65] L. Scapoli, A. Palmieri, L. Lo Muzio et al., "MicroRNA expression profiling of oral carcinoma identifies new markers of tumor 
progression," International Journal of Immunopathology and Pharmacology, vol. 23, no. 4, pp. 1229-1234, 2010.

[66] C. B. Lajer, F. C. Nielsen, L. Friis-Hansen et al., "Different miRNA signatures of oral and pharyngeal squamous cell carcinomas: a prospective translational study," British Journal of Cancer, vol. 104, no. 5, pp. 830-840, 2011. 

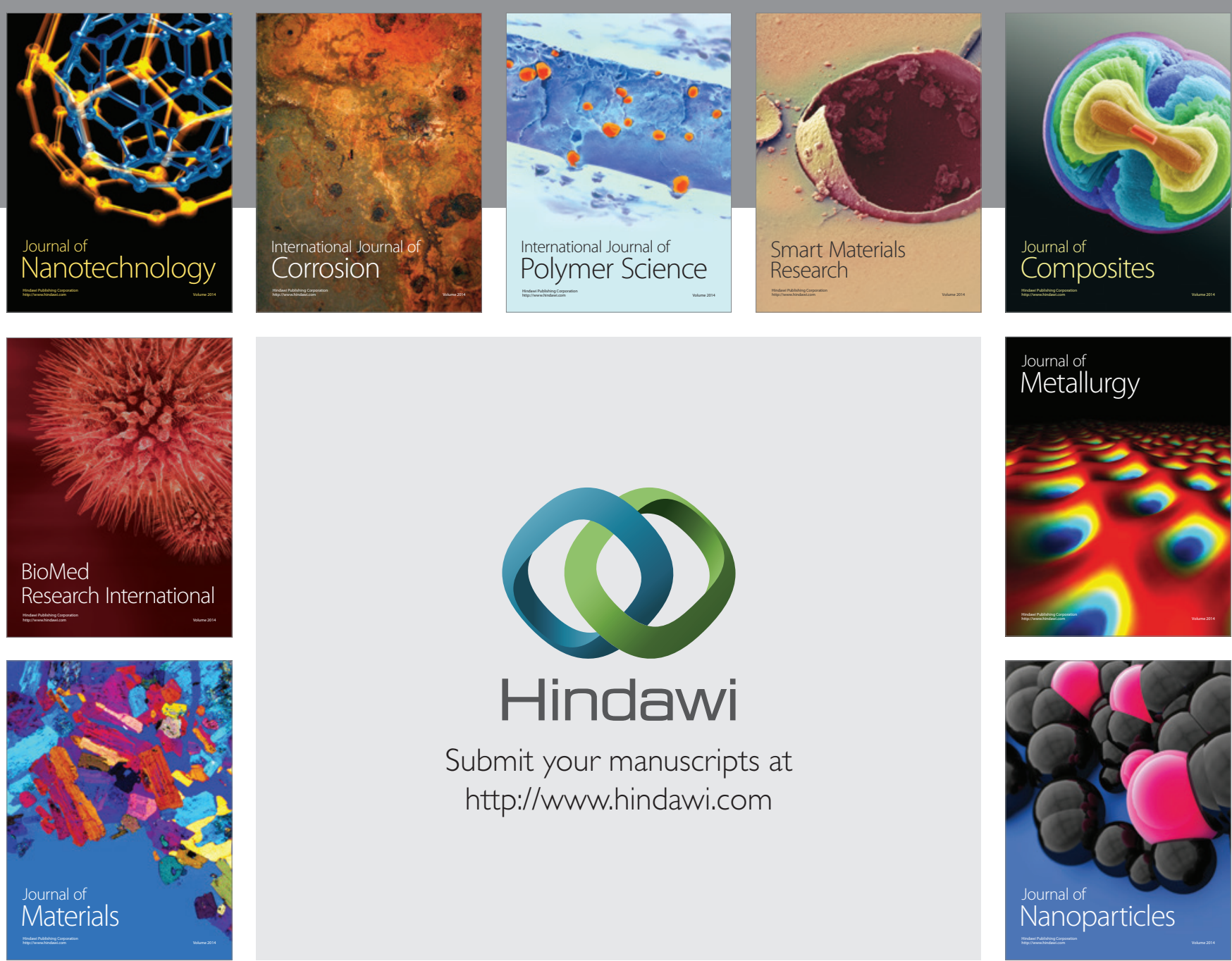

\section{Hindawi}

Submit your manuscripts at

http://www.hindawi.com

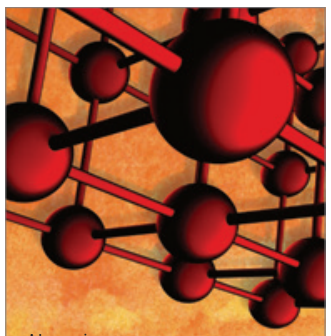

Materials Science and Engineering
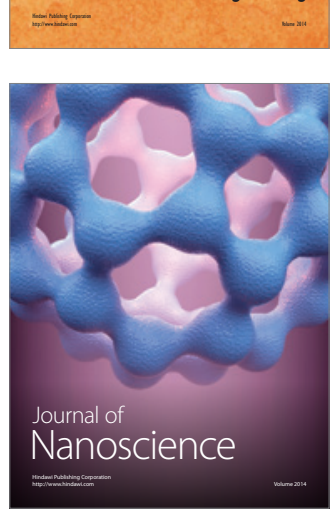
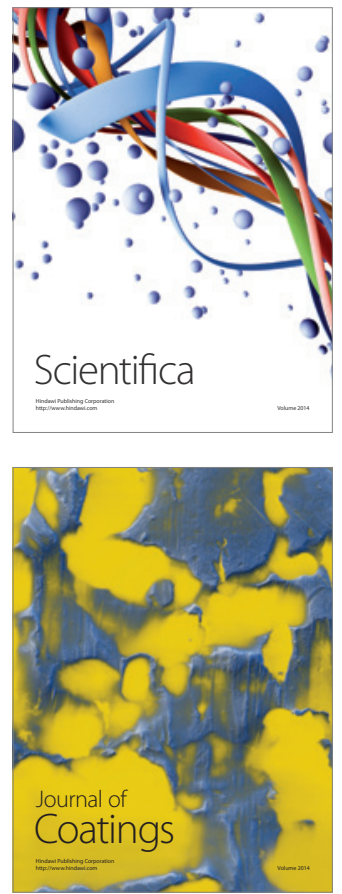
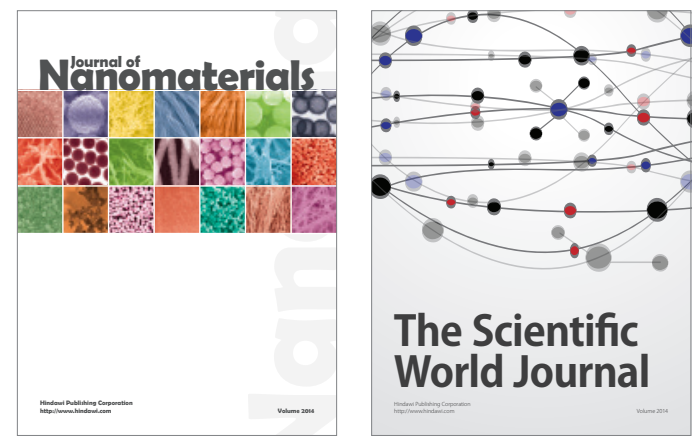

The Scientific World Journal
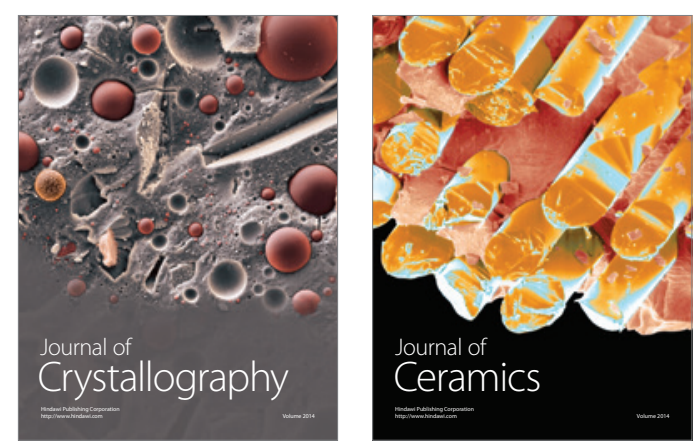
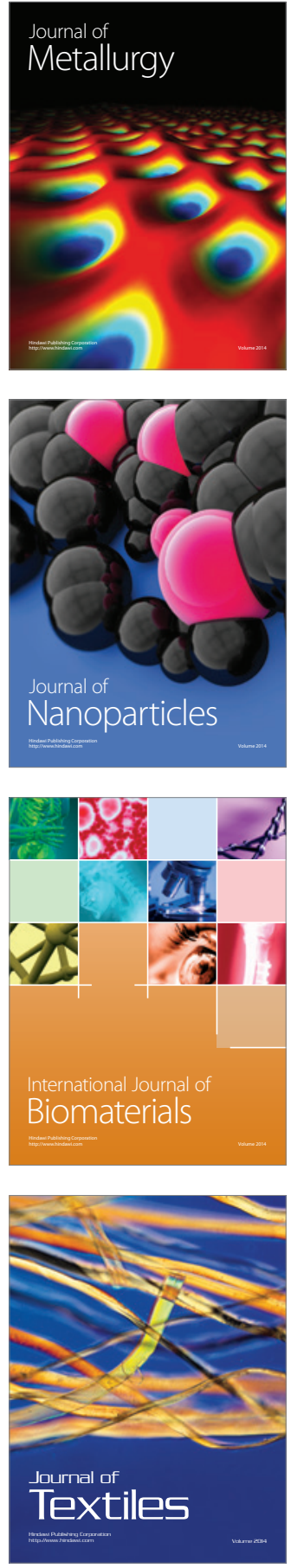